Noname manuscript No.

(will be inserted by the editor)

\title{
Retrospective Evaluation and SEEG Trajectory Analysis for Interactive Multi-trajectory Planner Assistant
}

\author{
Davide Scorza - Elena De Momi • Lisa \\ Plaino - Gaetano Amoroso - Gabriele \\ Arnulfo - Massimo Narizzano · Luis \\ Kabongo - Francesco Cardinale
}

Received: date / Accepted: date

\section{Abstract}

Purpose: Focal epilepsy is a neurological disease that can be surgically treated by removing area of the brain generating the seizures. The stereotactic electroencephalography (SEEG) procedure allows patient brain activity to be recorded in order to localize the onset of seizures through the placement of intracranial electrodes. The planning phase can be cumbersome and very time consuming, and no quantitative information is provided to neurosurgeons re-

\footnotetext{
D. Scorza

e-Health and Biomedical Applications Department, Vicomtech-IK4, Donostia-San Sebastián, Spain and

Dipartimento di Elettronica, Informazione e Bioingegneria (DEIB), Politecnico di Milano, Milan, Italy and

Biodonostia Health Research Institute, Donostia-San Sebastián, Spain Tel.: +34-943309230

E-mail: dscorza@vicomtech.org

E. De Momi, L. Plaino, G.Amoroso

Dipartimento di Elettronica, Informazione e Bioingegneria (DEIB), Politecnico di Milano, Milan, Italy

E-mail: $\quad$ elena.demomi@polimi.it, lisa.plaino@mail.polimi.it ， gaetano.amoroso@mail.polimi.it,

G. Arnulfo, M. Narizzano

Department of Informatics, BioEngineering Robotics and System engineering (DIBRIS),

University of Genoa, Genova, Italy

E-mail: gabriele.arnulfo@gmail.com,massimo.narizzano@unige.it,

L. Kabongo

e-Health and Biomedical Applications Department, Vicomtech-IK4, Donostia-San Sebastián, Spain and

Biodonostia Health Research Institute, Donostia-San Sebastián, Spain E-mail: lkabongo@vicomtech.org

F. Cardinale

Claudio Munari Centre for Epilepsy and Parkinson surgery, Niguarda Ca' Granda Hospital, Milan, Italy E-mail: francesco.cardinale@ospedaleniguarda.it
} 
garding the safety and efficacy of their trajectories. In this work, we present a novel architecture specifically designed to ease the SEEG trajectory planning using the 3D Slicer platform as a basis. Methods: Trajectories are autoo matically optimized following criteria like vessel distance and insertion angle. Multi-trajectory optimization and conflict resolution is optimized through a selective brute force approach based on a conflict graph construction. Additionally, electrode-specific optimization constraints can be defined, and an advanced verification module allows neurosurgeons to evaluate the feasibility

25 of the trajectory. Results: A retrospective evaluation was performed using manually planned trajectories on 20 patients: the planning algorithm optimized and improved trajectories in $98 \%$ of cases. We were able to resolve and optimize the remaining $2 \%$ by applying electrode specific constraints based on manual planning values. In addition, we found that the global parameters used 30 discards $68 \%$ of the manual planned trajectories, even when they represent a safe clinical choice. Conclusions: Our approach improved manual planned trajectories in $98 \%$ of cases in terms of quantitative indexes, even when applying more conservative criteria with respect to actual clinical practice. The improved multi-trajectory strategy overcomes the previous work limitations, 35 and allows electrode optimization within a tolerable time-span.

Keywords SEEG · Automated Planning · Computer Assisted Surgery · Image Guided Surgery · Epilepsy

\section{Introduction}

Epilepsy affects about 50 million people, $\sim 60 \%$ of these suffer partial seizures 40 and $\sim 25 \%$ of these are medically refractory to antiepileptic drug treatments, and are therefore potential candidates for surgery $[27,13]$. The aim of the surgery is the resection or disconnection of the Epileptogenic Zone (EZ), defined as "site of the beginning and of primary organization of the epileptic seizures" [22]. As previously reported, in $25 \%$ to $50 \%$ of subjects, identifica-

45 tion of the EZ entails the use of intracranial electroencephalography recordings [8]. StereoElectroEncephaloGraphy (SEEG) is a methodology originally developed by Bancaud and Talairach [2]. It consists of placing a number of multilead intracerebral electrodes for a three-dimensional (3D) investigation aimed at locating the EZ. Although the main principles of the surgery have not 50 changed, the development of modern Computer Assisted Surgery (CAS) and Image Guided Surgery (IGS) systems have modernized the workflow. The aim of updated surgical procedures is to increase patient safety, treatment efficacy, and reduce intervention time [6].

The correct positioning of intracerebral electrodes must accomplish the ac55 curate targeting of the desired intracerebral structures while minimizing the risk of complications, therefore SEEG trajectory planning is crucial. Accordingly, some of the criteria that have to be taken into account are the maximization of the distances to vessels and from sulcal entry, the minimization of the insertion angle of electrodes with respect to the skull normal and the avoidance 
60 of some structures such as the largest chambers of the lateral ventricles. Not satisfying one of these constraints could lead to dangerous complications such as bleeding, infections, cerebrospinal fluid leakage or electrode deviations that could result in serious injuries or even death. Traditionally, SEEG planning is performed by a neurosurgeon, who manually selects the entry points (EP) and

65 the target points (TP) by visually inspecting multi-modal images like Magnetic Resonance Imaging (MRI), Fluoro-D-Glucose-Positron-Emission-Tomography (FDG-PET) and angiographic datasets, etc ... [25]. Due to the usual amount of electrodes (up to 18 electrodes per hemisphere) and the need for high accuracy, the planning procedure is complex and time consuming (2-3 hours per procedure). Automated computer assisted planning may significantly reduce planning time and provide quantitative information about the safety and efficacy of trajectories. Specific constraints adapted to patient's anatomy can be modeled from clinical images, and serve as basis for the optimization of trajectories.

Different automatic algorithms have been proposed for minimally invasive neurosurgery, mainly for SEEG, Deep Brain Stimulation (DBS) and needle biopsies. The ultimate goal is to assist surgeons during the planning phase and find feasible and safe trajectories according to the application constraints. In [18], the authors presented a biopsy planner to reach deep seated brain tumors, which interactively assists surgeons during manual trajectory assessment through the interactive visualization of trajectory risks. Another group $[29,30]$ proposed a method which assigns risk values to each segmented critical structure based on the estimated damage that could be caused by crossing them. Results are visualized as color maps, and risk cards associated to trajectories 85 provide quantitative information when making the final choice. An alternative method was presented in [24], which generated access maps to guide surgeons during the selection of an entrance point to access specific regions and target anatomical structures. In [26], the estimated risk associated to accessing path was visualized interactively thanks to GPU optimized methods.

$90 \quad$ Other approaches automatically compute trajectories and select the best set based on the maximization of different constraints. In [15], the authors proposed a hybrid approach for DBS planning by providing information in the form of a color map and then automatically proposing a trajectory plan. Similarly, other authors $([4,3,5,21,20])$ implemented approaches for DBS which

95 take into account different restrictions as vessels and sulci avoidance, precision on target, ventricle avoidance, etc.

In relation to SEEG planning, in [12], the authors presented a multitrajectory automated planner which optimizes electrode trajectories and operates serially in the event of electrode conflicts, making the final plan biased 100 by the electrode optimization order. In [11] they overcame this problem by computing all possible combinations of electrode configurations. However, this approach is computationally inefficient, requiring too much time or forcing the user to decrease the number of solutions to be taken into account. Another implementation was presented in $[31,32]$, where the authors developed an automated MT planning algorithm that considers the distance to criti- 
cal structures, trajectory length, insertion angle, gray matter (GM) ratio and the interference between trajectories. Dynamic programming and a depth first search algorithm speed up the search strategy, leading to an optimal plan which takes into account all electrode positions simultaneously. In [33], the 10 authors centered the study on the localization of the electrode contacts in order to maximize the coverage of the region of interest.

Most of the presented methods are based on constraints which have been defined beforehand in collaboration with surgeons and medical staff. Moreover, these parameters are usually applied to all the trajectories that have to be 15 optimized, without considering different values based on additional factors such as the anatomical region explored. To the best of our knowledge, only in [14] the authors have retrospectively analyzed the planned trajectories to define the optimal weights for their cost function. A retrospective quantitative analysis of manually planned trajectories was performed in order to better understand routine clinical practice. Parameters have been collected and stored on a database and these will be used to improve the optimization constraints. Furthermore, we present an improved planning assistant built in 3D Slicer 4.5.0-1 [19] which improves the functionalities described in previous works $[12,11]$. This planner version allows the optimization of electrode trajectories 125 based on the following constraints:

1. Vessel avoidance

2. Sulcus avoidance

3. Insertion Angle with respect to the skull surface

4. Initial entry and target brain region definition

5. Electrode conflicts avoidance by means of a selective brute force approach

With respect to our previous work, we introduced two main innovations. The first one is that an atlas is used to limit our search space to the anatomical structures in which the neurosurgeon placed EPs and TPs. This feature is fundamental in order to respect the epileptological strategy of the implantation. The second new development is that we have overcome the computational limitations which affected our previous version in the case of electrode conflicts. Instead of considering all possible combinations of trajectories, a new selective brute force approach based on a graph construction was implemented. This method allowed us to run our optimization algorithm in a reasonable time using 3D Slicer platform which, as far as we know, does not natively offer the possibility of multi-core parallelization or GPU acceleration.

Our work is structured as follows: section 2 presents the implementation details and the retrospective validation. A description of each planner module is provided, with an explanation of its usage and the optimization strategy applied. Subsequently the characteristics of the experiment performed on retrospective patient data, illustrating methods, images used and indexes that have been evaluated are given in 2.4. Section 3 presents the results of the retrospective validation on 20 patients, for a total of 253 trajectories and compares the Manual Planning (MP), Optimized Single Trajectory Planner (OSTP) to the 
Optimized Multi-Trajectory Planner (OMTP) strategy. Finally a discussion and description of future work is provided in section 4 .

\section{Materials and Methods}

The SEEG-Planner extension built using 3D Slicer is composed of three different modules, each providing a different interaction level and assisting surgeons during a specific phase of the planning procedure. The Image Processing Module (section 2.1) allows the user to load and interactively prepare patient images required during the optimization procedure. The Optimization Module (section 2.2) enables neurosurgeons to select desired entry and target brain regions to initialize electrode trajectories and automatically computes the optimal ones according to chosen constraints. Finally, in the Advanced Trajectories Verification Module (section 2.3) surgeons can assess the surgical feasibility of the proposed plan. This module simplifies this phase with advanced interactive tools for vessel enhancement visualization and provides additional quantitative information about vessels distance.

Once a plan has been defined, information and data on the trajectories are stored in a trajectory database containing relevant information (recorded brain zones, parameter values, etc ...) which will be used for further studies.

\subsection{Image Processing Module}

This module initializes the patient planning procedure by loading the required images in 3D Slicer and generating additional data which will be used during the trajectory optimization. The modules uses 4 main inputs:

- CT-Angiography Volume

- Brain cortical surface mesh

- Curvature data relative to the cortical mesh

175 - CT acquisition of head bone or, alternatively, a T1-MRI

First, the user interactively selects a global threshold to segment vessels, visually guided by a volume-rendered output in the $3 \mathrm{D}$ view (Figure 1b). The volume is then visually inspected and once the surgeon finds a satisfactory result, a distance map is computed using Danielsson's distance mapping algorithm [10]. Second, the user defines a cortical curvature threshold representing feasible cortical entry points. This threshold is applied to the curvature data and transformed into a new surface which defines whether each vertex represents a feasible or unfeasible entry point (Figure 1c). Generally speaking, the curvature, which represents the cortical folding, is an effective index to distinguish between gyrus and sulci. Finally, the user obtains the patient skull or skin surface by thresholding the CT-bone volume or the T1-MRI, respectively (Figure 1d).

In our workflow, we used a Cone-Beam CT (CBCT) mobile scanner to obtain a $3 \mathrm{D}$ catheter angiography. Prior to administering the contrast medium 
injection, a preliminary CT volume (CT-bone) is acquired to obtain a bonemask that will be subtracted from the enhanced dataset, thus keeping only the vascular tree. Therefore, exploiting this 3D CBCT DSA approach, a single threshold approach is sufficient to construct a binary volume containing white voxels representing vessels and black for everything else. In order to obtain surface and curvature data we used Freesurfer (FS) [16], a well-established MRI imaging pipeline that has been proven to reliably segment cortical sheets [7]. It should be mentioned that any other program able to provide such information could be used as an alternative to FS. Additionally, we used the Destrieux Atlas [17] (Figure 1a) provided by FS as a probabilistic atlas which will be used during electrode optimization. We choose the Destrieux since it provides a finer representation of anatomical structures compared to Deskian-Killiany. Also in this case, the user can load his/her own preferred atlas.

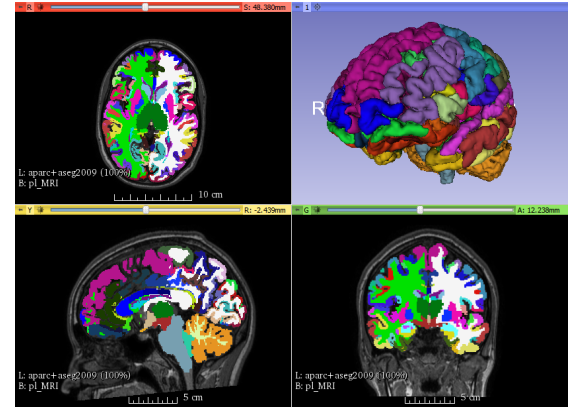

(a)

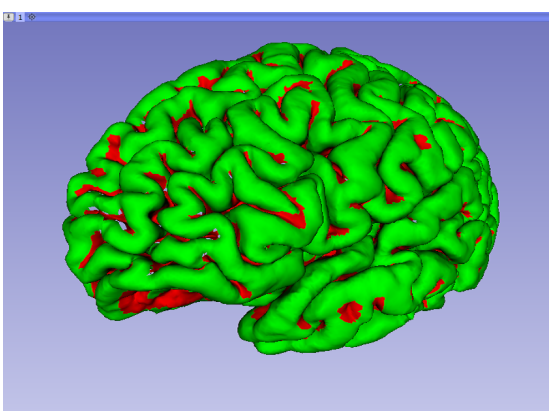

(c)

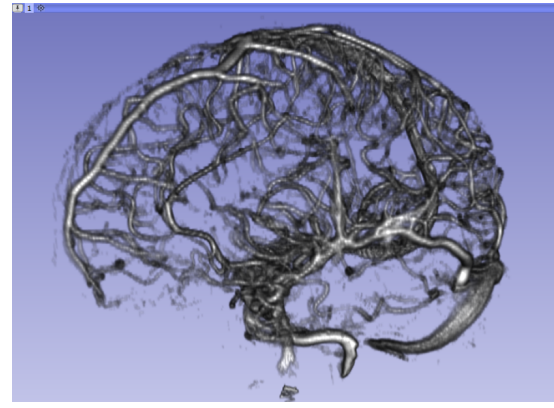

(b)

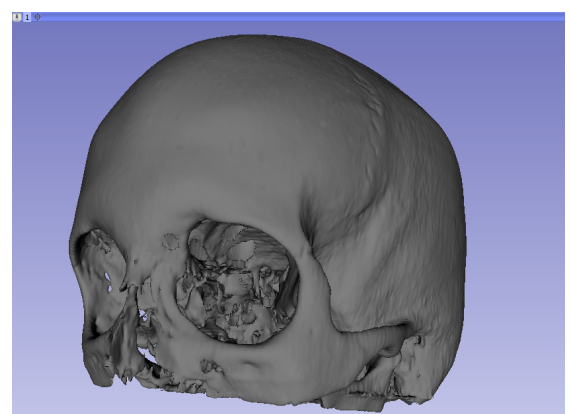

(d)

Fig. 1 Image Processing Module: (a) shows the Destrieux Atlas co-registered to MRI module during FS processing. (b) shows the volume rendering helping surgeons during the threshold selection for vessels binarization. (c) is the cortical surface divided into possible entry regions (green) and discarded regions (red). (d) is the skull surface obtained from CT acquisition. 


\subsection{Optimization Module}

The Optimization module attempts to find the best combination of electrode egy that has been planned. The implemented optimization approach is divided into four steps. The first (i) aims to define a set of feasible EP/TP. Subsequently, (ii) we optimize single trajectories constructed by pairing a feasible entry and target points for each electrode. Finally, we (iii) construct a conflict found.

Initially, the user defines a set of EP and TP to represent the desired intracerebral investigation strategy. These points represent the initial 3D coordinates of the seed points that will be used later in the optimization. By defining these based on the atlas used (i.e. Destrieux atlas). As an initial output, the module provides a suggestion for electrode models based on the total electrode distance measured as the Euclidean distance from EP to TP. In our centre we use Microdeep ${ }^{\circledR}$ (DixiMedical, Besançon, France) or Depth Electrodes Range (Alcis, Besançon, France). However, we provide a configuration file in json format which contains the geometric information of the electrode models and where it is possible to add other models from different manufacturers.

Subsequently, for any given electrode, the module constructs all possible trajectories starting from the user selected EP/TP pair. For any given pair, the module defines a circular or spherical Region Of Interests (ROI) for EP and TP with a radius of $r_{E P}$ and $r_{T P}$, respectively. Then, we map all possible entry and target points to the corresponding closest voxel coordinates. We discard all those points contained in the circular ROI that are considered unfeasible based on the curvature surface built during the initialization module as EP candidates. Furthermore, we discard all those voxels which do not belong to the corresponding initial anatomical region selected by the user as EP and TP candidates. The collections of all feasible EP and TP among the possible candidates constitute the accepted EP $\left(S_{E P}\right)$ and TP $\left(S_{T P}\right)$ that will be used for optimization.

The optimization strategy is based on four hard constraints that have to be satisfied in order to find the best combination of trajectories. Hence, (1) we define $\theta_{\max }$ as the maximum insertion angle with respect to skull normal acceptable for electrode placement. Regarding vessel distance (2), we split each trajectory into two tracts. Therefore, the user defines a length value $\zeta$ applied from the cortical EP to separate the cortical and distal tract and a distance threshold value of $\delta^{E P}$ representing the minimum distance from the closest vessel which has been applied to the first tract of the trajectory (Figure 2). The distance from vessels can be relaxed while proceeding in depth. Thus, a different threshold $\delta$ has to be defined for the second tract, usually satisfying $\delta^{E P} \geq \delta$. This approach arises from the consideration that the first tract, closer to the cortical surface, represents the zone where skull drilling and dura mater ablation are performed. Therefore, safety issues require a higher distance 
between the trajectory and the closest vessel in that zone while, nearer to TP, this constraint can be relaxed considering that SEEG electrodes are essentially atraumatic [8]. The user is allowed to change this parameter in the case of more traumatic devices.

Finally, we define $\gamma_{\min }$ as the minimum distance (3) between electrodes not causing any conflict and (4) the weights $\omega_{v}$ and $\omega_{a}$ for the final cost function 4 . The default values applied are shown in table 1. A resolution value of $\xi=0.25 \mathrm{~mm}$ for sampling the trajectories during the optimization procedure was defined according to images resolution and neurosurgeons suggestions.

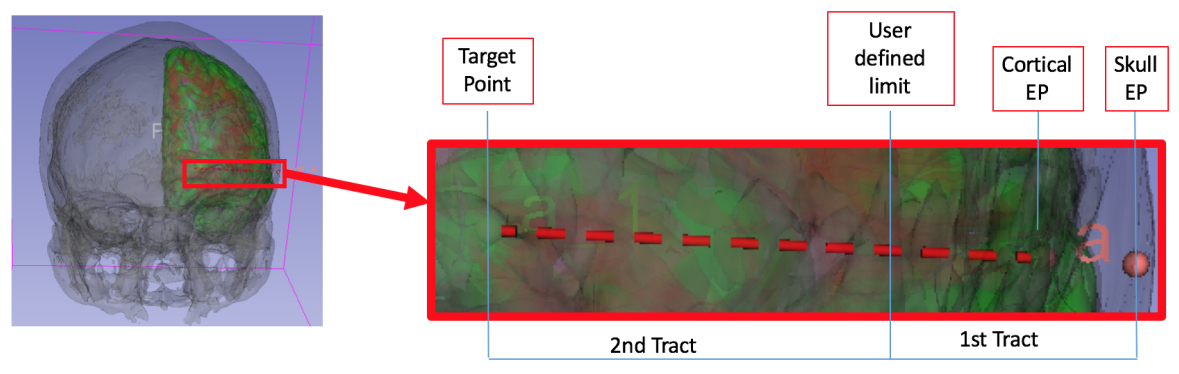

Fig. 2 Electrode trajectory tracts: in the first tract, a stronger constraint to vessel distance is applied, but this is relaxed reaching the TP. Both values can be manually adjusted.

Table 1 Default values applied for optimization parameters

\begin{tabular}{lc|lc}
\multicolumn{1}{c}{ Hard constraints } & \multicolumn{2}{c}{ Optimization Values } \\
\hline Vessel distance first Tract $[\mathrm{mm}]$ & 4 & EP search radius $[\mathrm{mm}]$ & 7 \\
Vessel distance second Tract $[\mathrm{mm}]$ & 1 & TP search radius $[\mathrm{mm}]$ & 3 \\
Insertion Angle $[\mathrm{deg}]$ & 30 & First tract Length $[\mathrm{mm}]$ & 10 \\
Inter-electrodes minimum distance $[\mathrm{mm}]$ & 3 & $\omega_{v}, \omega_{a}[12]$ & $0.8,0.2$
\end{tabular}

Finally, we run the MT strategy in two phases, starting with a dense search around the user seed points and applying a selective brute force algorithm to avoid conflicts. For each electrode, the total number of possible trajectories $M$ is represented by the combination of all possible EPs and TPs (equation 1).

$$
M=\left|S_{E P}\right| *\left|S_{T P}\right|
$$

where symbols $\left|S_{E P}\right|$ and $\left|S_{T P}\right|$ represent the cardinality of each collection of points.

the insertion angle $\theta(j)$ is computed with respect to the skull normal, with $j=1,2, \ldots, M$ and a cost function $f_{\theta}(j)$ is computed:

$$
f_{\theta}(j)= \begin{cases}\text { discarded if } & \theta(j)>\theta_{\max } \\ \frac{\theta_{\max }-\theta_{j}}{\theta_{\max }} & \text { otherwise }\end{cases}
$$


A higher score is given to those trajectories with a smaller angle with respect to skull normal.

The cost function for vessel distance $f_{v}$ is computed for each trajectory, based on the distance map generated in the previous module (2.1). The trajectory length $\operatorname{len}(j)$ is defined by the Euclidean distance between $E P_{\text {skull }}(j)$ and $T P(j)$, and trajectory $j$ is divided into a set of control points $N=\frac{\operatorname{len}(j)}{\xi}$. The corresponding value on the distance map is checked for each control point, which represents the distance from the closest vessel $d_{v}$, and the cost function is computed as following:

$$
f_{v}(j)= \begin{cases}\text { discarded } & \text { if } d_{v}(i)>\delta^{\text {thresh }} \\ \frac{1}{N+1} *\left(\sum_{i=1}^{N} \frac{d_{v}(i)-\delta_{v}^{m i n}}{\delta_{v}^{m \text { max }}-\delta_{v}^{\text {min }}}+\frac{\delta_{v}^{\text {min }}-\delta^{\text {thresh }}}{\delta^{\text {thresh }}}\right) & \text { otherwise }\end{cases}
$$

where $i=1,2, \ldots, N$, $\delta^{\text {thresh }}$ is respectively $\delta^{E P}$ or $\delta$ based on the trajectory tract, $\delta_{v}^{\min , \max }$ are respectively the minimum and maximum distance value for trajectory $j$.

Finally, a total cost function is computed as a weighted sum between the two components of each possible trajectory $j$ :

$$
S(j)=\omega_{v} * f_{v}(j)+\omega_{a} * f_{\theta}(j)
$$

A recursive strategy is applied in case all trajectories are discarded, which increases iteratively the search radius at EP and TP and the optimization is restarted.

At this point, each electrode has a total number $A$ of acceptable trajectories that have been computed and sorted in descending order based on the cost function expressed in equation 4.

Then, to prevent conflicts, we define an undirected conflict graph $G=$ $(V, E)$ where each node represents an electrode with $A$ possible states corresponding to the accepted trajectories, and edges $E$ represent possible conflicts among them.

At initial state, no possible conflicts have been identified, therefore $G=$ $(V, \emptyset)$. Then, we compute the Euclidean distance $\gamma_{i, k}$ between nodes $v_{i}, v_{k}$ of the conflict graph considering their initial EP and TP. We establish a possible conflict (create edge $e_{l}$ between $v_{i}, v_{k}$ ) if:

$$
\gamma_{i, k}-\left(r^{i}+r^{k}\right)<\gamma_{\text {min }}
$$

where $r_{i}=\max \left(r_{E P}^{i}, r_{T P}^{i}\right)$ and $r_{k}=\max \left(r_{E P}^{k}, r_{T P}^{k}\right)$. This is equivalent to estimating the area in which electrodes might be placed using the optimization algorithm, and checking whether these areas overlap or have a minimum distance inferior to $\gamma_{\min }$. Notice that the existence of an edge $e_{l} \in E$ represents a candidate conflict between the nodes $v_{i}, v_{k} \in V$. Later, it will be verified if a conflict actually exists between $v_{i}, v_{k}$. Theoretically, by applying this criterion, there is the possibility to obtain a fully connected graph in which all electrodes mutually influence their final positions. In that case, all possible 
combinations of all electrode trajectories computed need to be analyzed as in [11]. However, it represents an unlikely situation in which the electrodes are all close to each other. From a clinical point of view, there is no point in placing all the electrodes in a restricted brain zone, since the aim of the surgery is to explore brain activity find the epileptogenic focus. Therefore, we assume such a situation as highly improbable.

A more realistic scenario is to obtain a disconnected graph, in which it is possible to identify one or more subgraphs $G[S] \subseteq G=(V, E)$ comprising connected nodes. Then, we traverse each subgraph checking for conflicts by computing the Euclidean distance between the final position of the electrode pairs in the subgraphs. If a conflict is found, we take into account all possible combinations of electrode trajectories $I=A_{1} * A_{2} * \ldots * A_{\mid G[S]} \mid$ present in the subgraph to find the best conflict-free combination of electrode trajectories in the subgraph. For each combination $q \subseteq I$ it is possible to compute a new cost function $\Gamma(q)$, which evaluates the best combination of electrode trajectories contained in the subgraph $G[S]$ which avoids conflicts:

$$
\Gamma(q)=\frac{(1)}{|G[S]|} * \sum_{h=1}^{|G[S]|} S_{h}(j)
$$

where $j=1,2, \ldots, A$ and $A$ depends on the number of possible states of each node of the subgraphs. Finally, the combination of trajectories which maximize the cost function 6 is selected:

$$
\Gamma=\max _{q} \Gamma(q)
$$

In this way, we are able to split the conflict resolution into smaller subproblems that can be independently analyzed, reducing the time and computational resources required to determine valid solutions, as compared to complete brute-force exploration.

In addition, it should be highlighted that the use of the graph does not imply any degradation of results with respect to a standard brute-force approach. To demonstrate this point, consider a case study of $n=3$ electrodes $e_{1}, e_{2}, e_{3}$ where $\gamma_{1,2}-\left(r_{1}+r_{2}\right)<\gamma_{\text {min }}$, therefore causing a possible conflict between $e_{1}, e_{2}$. Once the three electrodes have been optimized, we obtain a pool of possible solutions representing the cost function values of the possible states of each electrode $S_{1}(j), S_{2}(j), S_{3}(j)$. For simplicity, we assume that $j=1, \ldots, 5$ and then each electrode can have only $J_{\max }=5$ possible states that have been computed during the optimization and are ranked in descending order. Table 2 represents the possible states of each electrode of the case study.

Using the standard brute force approach, the conflict resolution method analyzes all possible trajectory combinations between all electrodes, for a total of $J_{\max }^{n}=5^{3}$ possible combinations. After traversing all the solution space, the combination of conflict-free trajectories which maximize the group cost function $\Gamma$ will be selected, $\Gamma=\frac{S_{1}(4)+S_{2}(3)+S_{3}(1)}{3}$. By using our approach (graph), only $e_{1}, e_{2}$ solution space is traversed, given that $e_{3}$ cannot cause any 
Table 2 Solution Space of case study. We report the possible state of each electrode after optimization. $\left(^{*}\right)$ represents the trajectories which creates a conflict. In bold, the best trajectory combination which does not cause any conflict.

\begin{tabular}{c|c|c}
$e_{1}$ & $e_{2}$ & $e_{3}$ \\
\hline$S_{1}(1)^{*}$ & $S_{2}(1)^{*}$ & $\boldsymbol{S}_{\mathbf{3}}(\mathbf{1})$ \\
\hline$S_{1}(2)^{*}$ & $S_{2}(2)^{*}$ & $S_{3}(2)$ \\
\hline$S_{1}(3)^{*}$ & $\boldsymbol{S}_{\mathbf{2}}(\mathbf{3})$ & $S_{3}(3)$ \\
\hline $\boldsymbol{S}_{\mathbf{1}}(\mathbf{4})$ & $S_{2}(4)$ & $S_{3}(4)$ \\
\hline$S_{1}(5)$ & $S_{2}(5)$ & $S_{3}(5)$
\end{tabular}

In order to allow surgeons to evaluate the feasibility of the proposed trajectories, we propose an advanced module for their visualization and analysis. The user can select an electrode and apply a probe eye view, which allows the visualization on the plane perpendicular to the electrode trajectory on the original angiography dataset. In addition, a volume portion can be selected to generate Maximum Intensity Projection (MIP) images on that plane and enhance vessel visualization, especially in images with contrast medium (Figure 3). Finally, in order to reduce background noise, an automatic vessel segmentation based on Gaussian Mixture Model (GMM) and Markov Random Field (MRF) can be applied directly to the MIP image, in order to identify only vessels around the electrode [28].

\subsection{Retrospective Evaluation}

In order to determine if our approach would adapt to real clinical practice we conducted a preliminary evaluation on 20 patients in the form of a retrospective analysis. We collected trajectories that had been planned manually by neurosurgeons and used them as seed points for our optimization. Since these trajectories represented the neurosurgeon's solution and no complications have been reported, we also extracted quantitative information to better understand the viability of our constraints. These data have been stored in a 


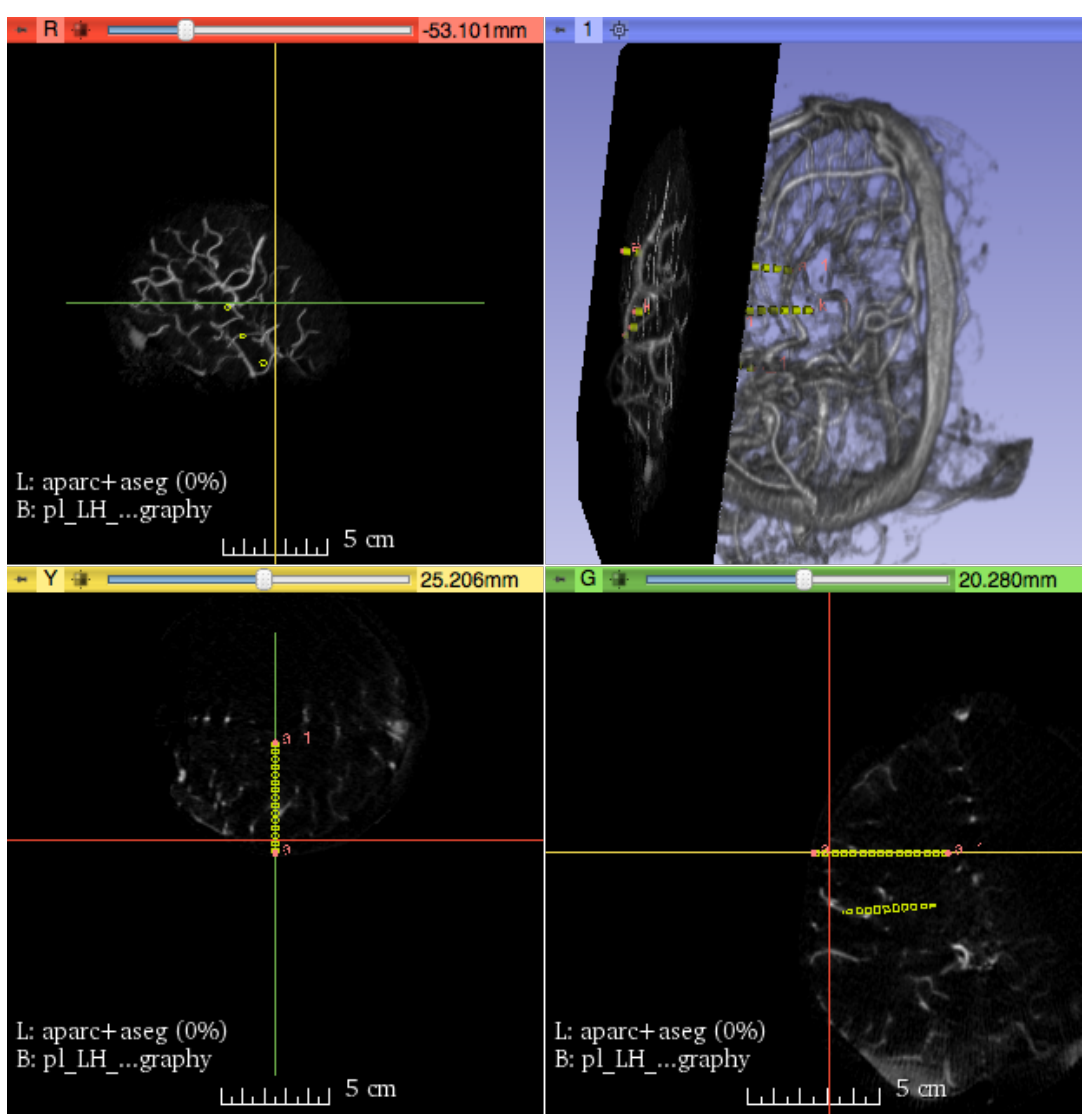

Fig. 3 Advanced electrode trajectory check with MIP image projection on Probe Eye View. The red slice view shows the plane perpendicular to the electrode on which the MIP has been applied. The other two views (yellow and green) show the two planes orthogonal to the electrode trajectory. These two, jointly with the slice intersection, help the neurosurgeon to understand the view position in relation to the electrode total length. Electrode models are enlarged to allow an easier visualization (yellow contacts).

connected database and will be used for future developments. Hence, we collected a total number of 253 trajectories, and compared the manual planning to the optimized MT strategy and the single trajectory strategy. Even though the planner allows electrode-specific constraints to be selected, in this particular preliminary study we used the same values for all electrodes as a global optimization strategy, as would have been performed at that time.

\subsubsection{Experimental Protocol}

Retrospective validation was performed on patients who underwent the SEEG procedure at "Cladio Munari" Centre for Epilepsy and Parkinson Surgery A.O. Ospedale Niguarda Ca Granda, Milan, Italy. No complications have been reported. For each patient, MR images were acquired using the hospital system 
1.5T (Intera Achieva, Philips Medical System, The Netherlands, T1 3D FFE sagittal images, $0.90 \mathrm{~mm} \times 1.07 \mathrm{~mm} \times 0.90 \mathrm{~mm}$ voxel dimensions, without any inter-slice gap, then reconstructed and reformatted on the axial plane with $560 \times 560 \times 220$ matrix, $0.45 \mathrm{~mm} \times 0.45 \mathrm{~mm} \times 0.9 \mathrm{~mm}$ voxel dimensions $). \mathrm{FS}$ pipeline was run to obtain the cortical reconstructed surface and the other structures explained previously. Angiographic images were acquired (O-arm, Medtronic Inc., US, $512 \times 512 \times 192,0.4 m m \times 0.4 m m \times 0.8 m m$ voxel) using contrast medium. Bone mask was removed following the procedure presented in [9]. Electrode trajectories were manually planned by neurosurgeons using the Voxim (IVS Technology GmbH, Chemnitz, Germany) software application. All previous steps were done specifically for the use of the automated planning assistant developed but already part of Niguarda Hospital Workflow for SEEG procedures. An experienced neurosurgeon performed the segmentation steps for each patient following the image processing module (section 2.1), and saved the results. Subsequently, vessel tree segmentation, possible entry regions and the skull surface have been generated and stored for the optimization step. The threshold values chosen for optimization were defined equally for all electrodes (table 1). We compared the performances of three methods: Manual Planning (MP), Optimized Single Trajectory Planning (OSTP), and Optimized MultiTrajectory Planning (OMTP). The difference between OSTP and OMTP was the management of inter-electrode conflicts: in OSTP, the electrodes that cause a conflict were not optimized and a warning message was displayed to the user. In order to quantitatively compare the methods, we proceeded as follows. For each trajectory and method we quantified the minimum distance from vessels in the first (1) and second tract (2) along with the insertion angle in relation to the skull normal (3). We defined a trajectory to be correctly planned if all the hard constraints (see 2.2) had been satisfied. Then, we quantified the trajectory success rate defined as the number of correct trajectories out of the total number. Furthermore, to evaluate the effects of the optimization approaches, we quantitatively evaluated the indexes defined above for the final electrode positions proposed by OMTP and OSTP in relation to MP trajectories. We performed a Kolmogorov-Smirnov test to check the normality of the computed indexes and, consequently, a two-sample t-test was performed to check for statistical differences. Statistical analysis was performed in Matlab. Finally, 415 we reported the computation time and the number of trajectories for each electrode that had been taken into account during the optimization procedures (section 3.3). To quantify the effect, in terms of computational efficiency, we estimated the number of trajecory combinations analyzed by the selective brute force approach compared to a classical brute force algorithm, as proposed

420 in [11]. Data has been processed on a laptop Acer v5-573G, Intel(R) Cose(TM) i7-4500U CPU @ 1.80GHz with 8GB of RAM DDR3 running Windows 10. 


\section{Results}

\subsection{Trajectory Success Rate}

Our algorithm discarded the majority of the manually planned trajectories by analyzing them without performing any optimization (table 3). Almost 50\% of them reported a minimum $\delta_{E P}$ inferior to the threshold applied. Additional experiments showed that by relaxing the first tract threshold $\delta_{E P}=3.8 \mathrm{~mm}$, the MP discarded trajectories fell to a total of $41 \%$, half of them because of a too large insertion angle. As expected, OSTP and OMTP methods improved these trajectories by reaching a success rate of $92 \%$ and $98 \%$, respectively. The $5 \%$ difference represents the OMTP capacity to resolve inter-electrode conflicts.

Table 3 Discarded trajectories using our algorithm, based on hard constraints used and image processing steps performed, for each method and a total of 253 trajectories. *Trajectories rejected based on $\delta_{E P}$ criteria reduced to $8 \%$ by relaxing the threshold to 3.8 $\mathrm{mm}$

\begin{tabular}{c|c|c|c} 
& MP & OSTP & OMTP \\
\hline$\theta$ & $47(18 \%)$ & $5(2 \%)$ & $5(2 \%)$ \\
$\delta_{E P}$ & $92^{*}(36 \%)$ & & \\
\hline$\delta$ & $34(13 \%)$ & 0 & 0 \\
\hline Conflicts & 0 & $9(3 \%)$ & 0 \\
\hline \hline Total Discarded & $173(68 \%)$ & $13(8 \%)$ & $5(2 \%)$
\end{tabular}

\subsection{Quantitative Evaluation}

Figure 4 reports on the final values of the three indexes considered after run-

ning the two optimization procedures, OSTP and OMTP. Figure 4a shows a general improvement, where the majority of points lie under the bisector representing a decrease in the maximum insertion angle. On the contrary, in figures $4 \mathrm{~b}$ and $4 \mathrm{c}$ we want to maximize the distance from vessels, subsequently the improvement is represented by points above the bisector. This was not always true, especially for the minimum distance in the second tract $\delta$ (Figure 4b) However, it should be noted that all optimized trajectories reported values which satisfied the threshold limits. This was not always accomplished by MP trajectories, as in all three images it is possible to find initial values which exceeded the maximum/minimum thresholds. Table 4 reports mean and standard deviation values computed for the three methods and confirms the general improvement provided by the optimization of the MP trajectories. Accordingly, statistical differences have been found between MP and OSTP and between MP and OMTP. No statistical differences have been found between the two optimization methods, however we computed median and inter-quartile range values of the differences found between the alternative trajectories proposed 
by OMTP in relation to OSTP. For the three indexes, we found a minimum distance from vessels in the first tract of $0.46(1.06) \mathrm{mm}$, in the second tract of $0.3(0.4)$ and an insertion angle of 1.03(5.86) deg.

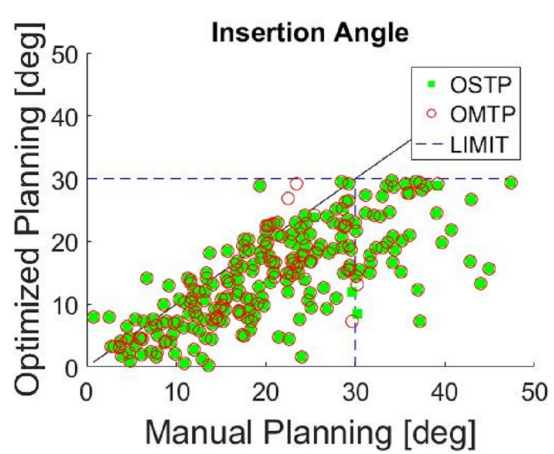

(a)

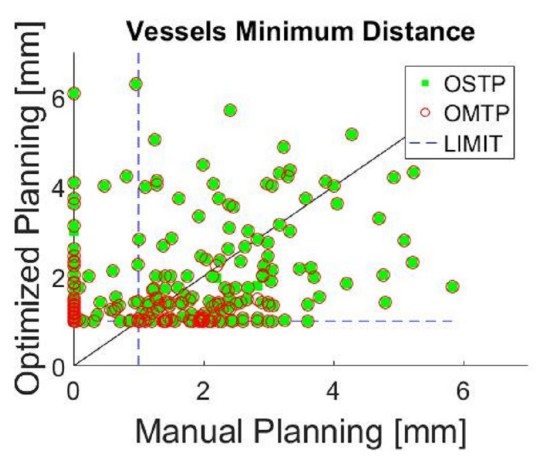

(b)

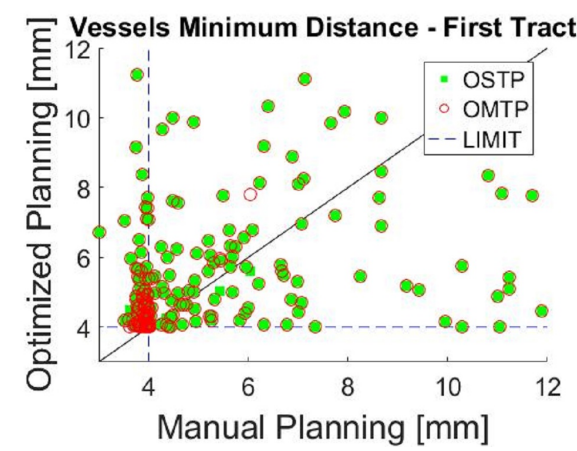

(c)

Fig. 4 Quantitative comparison of the three indexes computed in relation to the MP trajectories. $4 \mathrm{a}$ shows the values obtained from the insertion angle after the optimization procedures. The same is presented in $4 \mathrm{~b}$ and $4 \mathrm{c}$ for minimum vessel distance detected and minimum vessel distance in first tract respectively. On each graph, the x-axis reports the MP values, which represent the starting points, while y-axis reports the final values reached. The blue dashed lines represents the optimization hard constraints used in our algorithm.

Table 4 Mean and standard deviation values are reported for each method for the indexes computed.

\begin{tabular}{c|ccc} 
& MP & OSTP & OMTP \\
\hline$\theta[\mathrm{deg}]$ & $20.65 \pm 9.81$ & $14.44 \pm 7.54$ & $14.53 \pm 7.64$ \\
$\delta_{E P}[\mathrm{~mm}]$ & $4.78 \pm 3.55$ & $5.52 \pm 1.77$ & $5.54 \pm 1.78$ \\
$\delta[\mathrm{mm}]$ & $1.66 \pm 1.30$ & $1.96 \pm 1.35$ & $1.95 \pm 1.35$
\end{tabular}




\subsection{Computational Efficiency}

455 Regarding computational complexity, we report a mean processing time of $160.5 \pm 102.25$ seconds for a single electrode optimization. For these experiments, all the electrodes were optimized serially, making the total computational time strictly dependent on the electrodes number. For each electrode, entry and target point regions generated a mean number of $25 * 10^{3}$ possible

460 trajectories. During the optimization, the mean number of acceptable trajectories per electrode dropped to $2.7 * 10^{3}$. With regard to the conflicts graph, the largest subgraph built comprised 4 nodes, taking into account all patients. Then, in the case of conflicts, the total number of maximum combinations was reduced to an order of $10^{12}$, while with the previous method we would have

465 had a number of around $10^{41}$ (assuming a mean number of 12 electrodes in our patients). However, in the majority of the cases, the final electrode positions did not generate conflicts and the mean time used to traverse the conflict graph was in $0.015 \pm 0.005$ seconds.

\section{Discussion}

470 We have presented an improved automated multi-trajectory planner for SEEG, based on open source software widely used by the scientific community (3D Slicer, Freesurfer, FSL). Compared to our previous planner versions [12,11], the transformation into a multi-modular architecture improved modularity, flexibility and usability. An additional module implementing MIP images and 475 automatic vessel segmentation was presented, which simplifies the validation of proposed trajectories. Regarding optimization strategy, a dense search around EP and TP has been complemented with the use of an atlas, which restricts the search space to the anatomical regions identified by the initial EP and TP seeds. Compared to other groups $[32,33]$, the user initializes the electrodes by roughly selecting EP and TP. This is an easy and fast procedure but is closer to the clinical solution and limits exploration only on the surrounding area.

To guide the optimization procedure we defined global optimization parameters and hard constraints values according to surgeon experience and surgical constraints that can be found in literature $[11,32,33]$. However, the analysis of MP trajectories, according to the defined criterion, rejected $68 \%$ of them, even though those trajectories were used to perform safe implants without any complications reported. Furthermore, by lowering the threshold $\delta_{E P}$, we considerably reduced the number of MP discarded trajectories, suggesting the neurosurgeon's intention to follow the criterion strictly applied in the planner. Moreover, it must be considered that such trajectories have been planned with only visual assistance (3D images), without any quantitative information. These results suggest that the thresholds used in the optimization algorithm may need to be revised as it actually happens in real practice, when surgeons decide to be less restrictive in some cases. In addition, we cannot discard that new constraints have to be added to our algorithm, in order 
to take into account additional requirements that may influence the clinical choice. Nonetheless, OSTP and OMTP were able to optimize these trajectories, reaching a success rate of $93 \%$ and $98 \%$ respectively. The difference between the two methods underlies the importance of providing an efficient strategy able to manage inter-electrode conflicts. On the contrary, no possible solutions were found in 5 cases. When analyzing those patients' datasets, we found that the initial insertion angle of these MP trajectories was too large in relation to the maximum angle allowed, leading to the impossibility of finding a similar solution even for initialization methods which do not limit the search region. Therefore, as a result of the possibility to define electrode specific constraints, we increased the maximum angle allowed (40 deg) specifically for those electrodes. With this new constraint, the optimization method was able to find a feasible solution.

The quantitative analysis showed that both OMTP and OSTP were able to improve the MP trajectories used as initial seeds. When analyzing the graph presented in Figure 4a, the insertion angle has clearly been improved by both optimization methods, even when the initial values where further from the threshold defined. Figure $4 \mathrm{~b}$ reports a number of trajectories with initial 0 $\mathrm{mm}$ distance from vessels in the second tract. This effect is probably due to the threshold chosen during the vessel segmentation part. A too conservative threshold would include some noise which has been interpreted as small vessels by our procedure. However, the optimization strategy was able to find alternative paths and to provide final trajectories which complied with our hard constraints. Focusing on Figure $4 \mathrm{c}$, the high concentration of $\delta_{E P}$ initial values below, but in proximity of the defined threshold, confirms the neurosurgeon's intention to provide a minimum distance value around $4 \mathrm{~mm}$ in the first tract of the trajectories. This requirement has been satisfied by both optimization approaches. Lastly, since only 9 real inter-electrode conflicts have been detected, no statistical difference has been found between OMTP and OSTP. As ${ }_{525}$ expected, the OMTP strategy leads to alternative trajectories which preferentially increase the insertion angle rather than the minimum vessel distances, according to the weights expressed in the cost function (eq. 4). All the trajectories proposed satisfied the hard constraints applied in the optimization.

The multi-trajectory strategy presented and implemented in OMTP was able to resolve these inter-electrode conflicts and propose alternative solutions which satisfy all the constraints. The strategy implemented overcame the limitations of the previous multi-trajectory planner [11] by reducing the computational cost with a selective brute force approach. The graph construction allowed these electrodes to be split into independent subgroups (as reported in 5353.3 , the largest subgroup reported among all patients' trajectories comprised 4 electrodes), decreasing the computational effort by reducing the number of combinations to explore. This method allowed us to run our optimization algorithm in a reasonable time on the 3D Slicer platform that, to our understanding, does not offer the possibility of multi-core parallelization or GPU acceleration. 


\section{Conclusion}

We presented an automated multi-trajectory planner able to assist neurosurgeons during the different planning phases. We collected and analyzed in terms of quantitative indexes, 253 manually planned trajectories of patients who successfully underwent SEEG surgery. The optimization strategy allowed these indexes to be improved in $98 \%$ the cases by managing inter-electrode conflicts through an efficient selective brute force approach. The evaluated indexes showed an improvement in terms of safety compared to the MP trajectories, nonetheless, one of the major limitations of this work is the absence of a qualitative validation of the proposed new trajectories. In this regard, our architecture sets the basis for future developments and improvements to the different stages of the procedures and allows for future extended clinical trials and validation. In addition, future studies will focus on the analysis of MP trajectories to identify additional parameters that have not been included in our model yet and to select constraint values based on quantitative information. Finally, the use of the 3D Slicer platform as a development environment improves the planner flexibility and the possibility to integrate and modify the workflow according to custom tools developed at other centers. Future efforts will focus on the integration of SEEG tools such as the "seeg electroDE rEconstruction TOol" (DEETO) [1], and SEEG-Assistant [23] which allow the automatic segmentation of implanted electrodes from post-operative CT and offer other dedicated tools useful for viewing SEEG signals.

\section{Acknowledgement}

We are grateful to the three anonymous reviewers for their revisions and insightful comments.

\section{Conflict of Interest Statement}

The authors declare that they have no conflict of interest.

\section{Statement of Human Rights}

All procedures performed in studies involving human participants were in accordance with the ethical standards of the institutional and/or national research committee and with the 1964 Helsinki declaration and its later amendments or comparable ethical standards.

\section{Statement on Retrospective Studies}

Formal consent is not required for this type of study 


\section{Statement on Informed Consent}

Informed consent was obtained from all individual participants included in the study.

\section{Statement on Welfare of Animals}

This article does not contain any studies involving animals performed by any

of the authors.

\section{References}

1. Arnulfo, G., Narizzano, M., Cardinale, F., Fato, M.M., Palva, J.M.: Automatic segmentation of deep intracerebral electrodes in computed tomography scans. BMC bioinformatics 16(1), 1 (2015)

2. Bancaud, J.: La stéréo-électroencéphalographie dans l'épilepsie. Masson \& Cie Editeurs (1965)

3. Bériault, S., Al Subaie, F., Collins, D.L., Sadikot, A.F., Pike, G.B.: A multi-modal approach to computer-assisted deep brain stimulation trajectory planning. International journal of computer assisted radiology and surgery $\mathbf{7}(5), 687-704$ (2012)

4. Bériault, S., Al Subaie, F., Mok, K., Sadikot, A.F., Pike, G.B.: Automatic trajectory planning of DBS neurosurgery from multi-modal MRI datasets. In: Medical Image Computing and Computer-Assisted InterventionMICCAI 2011, pp. 259-266. Springer (2011)

5. Bériault, S., Drouin, S., Sadikot, A.F., Xiao, Y., Collins, D.L., Pike, G.B.: A prospective evaluation of computer-assisted deep brain stimulation trajectory planning. In: Clinical Image-Based Procedures. From Planning to Intervention, pp. 42-49. Springer (2012)

6. Cardinale, F., Casaceli, G., Raneri, F., Miller, J., Lo Russo, G.: Implantation of stereoelectroencephalography (seeg) electrodes: a systematic review. Clinical Neurophysiology pp. 490-502 (2016)

7. Cardinale, F., Chinnici, G., Bramerio, M., Mai, R., Sartori, I., Cossu, M., Russo, G.L., Castana, L., Colombo, N., Caborni, C., De Momi, E., Ferrigno, G.: Validation of freesurfer-estimated brain cortical thickness: comparison with histologic measurements. Neuroinformatics 12(4), 535-542 (2014)

8. Cardinale, F., Cossu, M., Castana, L., Casaceli, G., Schiariti, M.P., Miserocchi, A., Fuschillo, D., Moscato, A., Caborni, C., Arnulfo, G., Lo Russo, G.: Stereoelectroencephalography: surgical methodology, safety, and stereotactic application accuracy in 500 procedures. Neurosurgery 72(3), 353-366 (2013)

9. Cardinale, F., Pero, G., Quilici, L., Piano, M., Colombo, P., Moscato, A., Castana, L., Casaceli, G., Fuschillo, D., Gennari, L., Cenzato, M., Lo Russo, G., Cossu, M.: Cerebral angiography for multimodal surgical planning in epilepsy surgery: description of a new three-dimensional technique and literature review. World neurosurgery 84(2), 358-367 (2015)

10. Danielsson, P.E.: Euclidean distance mapping. Computer Graphics and image processing 14(3), 227-248 (1980)

11. De Momi, E., Caborni, C., Cardinale, F., Casaceli, G., Castana, L., Cossu, M., Mai, R., Gozzo, F., Francione, S., Tassi, L., Lo Russo, G., Ferrigno, G.: Multi-trajectories automatic planner for StereoElectroEncephaloGraphy (SEEG). International journal of computer assisted radiology and surgery 9(6), 1087-1097 (2014)

12. De Momi, E., Caborni, C., Cardinale, F., Castana, L., Casaceli, G., Cossu, M., Antiga, L., Ferrigno, G.: Automatic trajectory planner for StereoElectroEncephaloGraphy procedures: a retrospective study. Biomedical Engineering, IEEE Transactions on 60(4), 986-993 (2013) 
13. Dlugos, D.J.: The early identification of candidates for epilepsy surgery. Archives of neurology 58(10), 1543-1546 (2001)

14. Essert, C., Fernandez-Vidal, S., Capobianco, A., Haegelen, C., Karachi, C., Bardinet, E., Marchal, M., Jannin, P.: Statistical study of parameters for deep brain stimulation automatic preoperative planning of electrodes trajectories. International journal of computer assisted radiology and surgery 10(12), 1973-1983 (2015)

15. Essert, C., Haegelen, C., Lalys, F., Abadie, A., Jannin, P.: Automatic computation of electrode trajectories for deep brain stimulation: a hybrid symbolic and numerical approach. International journal of computer assisted radiology and surgery $\mathbf{7}(4), 517-$ $532(2012)$

16. Fischl, B.: Freesurfer. Neuroimage 62(2), 774-781 (2012)

17. Fischl, B., van der Kouwe, A., Destrieux, C., Halgren, E., Ségonne, F., Salat, D.H., Busa, E., Seidman, L.J., Goldstein, J., Kennedy, D., Caviness, V., Makris, N., Rosen, B., Dale, A.M.: Automatically parcellating the human cerebral cortex. Cerebral cortex 14(1), 11-22 (2004)

18. Herghelegiu, P., Manta, V., Perin, R., Bruckner, S., Gröller, E.: Biopsy planner-visual analysis for needle pathway planning in deep seated brain tumor biopsy. In: Computer Graphics Forum, vol. 31, pp. 1085-1094. Wiley Online Library (2012)

19. Kapur, T., Pieper, S., Fedorov, A., Fillion-Robin, J., Halle, M., O’Donnell, L., Lasso, A. Ungi, T., Pinter, C., Finet, J., Pujol, S., Jagadeesan, J., Tokuda, J., Norton, I., Estepar, R., Gering, D., Aerts, H., Jakab, M., Hata, N., Ibanez, L., D, B., Miller, J., Aylward, S., Grimson, W., Fichtinger, G., Wells, W., Lorensen, M., Schroeder, W., Kikinis, R.: Increasing the impact of medical image computing using community-based open-access hackathons: The na-mic and 3d slicer experience. Medical Image Analysis 33, 176-180 (2016)

20. Liu, Y., Dawant, B.M., Pallavaram, S., Neimat, J.S., Konrad, P.E., D'Haese, P.F., Datteri, R.D., Landman, B.A., Noble, J.H.: A surgeon specific automatic path planning algorithm for deep brain stimulation. In: SPIE medical imaging, pp. 83,161D-83,161D. International Society for Optics and Photonics (2012)

21. Liu, Y., Konrad, P.E., Neimat, J.S., Tatter, S.B., Yu, H., Datteri, R.D., Landman, B.A., Noble, J.H., Pallavaram, S., Dawant, B.M., DHaese, P.F.: Multisurgeon, multisite validation of a trajectory planning algorithm for deep brain stimulation procedures. Biomedical Engineering, IEEE Transactions on 61(9), 2479-2487 (2014)

22. Munari, C., Bancaud, J.: The role of stereo-electro-encephalography (seeg) in the evaluation of partial epileptic patients. In: The epilepsies, pp. 267-306. Butterworths New York (1987)

23. Narizzano, M., Arnulfo, G., Ricci, S., Toselli, B., Tisdall, M., Canessa, A., Fato, M.M. Cardinale, F.: Seeg assistant: a 3dslicer extension to support epilepsy surgery. BMC bioinformatics 18(1), 124 (2017)

24. Navkar, N.V., Tsekos, N.V., Stafford, J.R., Weinberg, J.S., Deng, Z.: Visualization and planning of neurosurgical interventions with straight access. In: Information Processing in Computer-Assisted Interventions, pp. 1-11. Springer (2010)

25. Nowell, M., Rodionov, R., Zombori, G., Sparks, R., Winston, G., Kinghorn, J., Diehl, B., Wehner, T., Miserocchi, A., McEvoy, A.W., Duncan, J.: Utility of 3d multimodality imaging in the implantation of intracranial electrodes in epilepsy. Epilepsia 56(3), 403413 (2015)

26. Rincn-Nigro, M., Navkar, N.V., Tsekos, N.V., Deng, Z.: GPU-accelerated interactive visualization and planning of neurosurgical interventions. Computer Graphics and Applications, IEEE 34(1), 22-31 (2014)

27. Rosenow, F., Lüders, H.: Presurgical evaluation of epilepsy. Brain 124(9), 1683-1700 (2001)

28. Scorza, D., Moccia, S., De Luca, G., Plaino, L., Cardinale, F., Mattos, L.S., Kabongo, L., De Momi, E.: Safe electrode trajectory planning in seeg via mip-based vessel segmentation. In: SPIE Medical Imaging, pp. 101,352C-101,352C. International Society for Optics and Photonics (2017)

29. Shamir, R.R., Joskowicz, L., Tamir, I., Dabool, E., Pertman, L., Ben-Ami, A., Shoshan, Y.: Reduced risk trajectory planning in image-guided keyhole neurosurgery. Medical physics 39(5), 2885-2895 (2012) 
30. Shamir, R.R., Tamir, I., Dabool, E., Joskowicz, L., Shoshan, Y.: A method for planning safe trajectories in image-guided keyhole neurosurgery. In: Medical Image Computing and Computer-Assisted InterventionMICCAI 2010, pp. 457-464. Springer (2010)

31. Sparks, R., Zombori, G., Rodionov, R., Nowell, M., Vos, S.B., Zuluaga, M.A., Diehl, B., Wehner, T., Miserocchi, A., McEvoy, A.W., Duncan, J., Ourselin, S.: Automated multiple trajectory planning algorithm for the placement of stereo-electroencephalography (seeg) electrodes in epilepsy treatment. International Journal of Computer Assisted Radiology and Surgery pp. 1-14 (2016)

32. Sparks, R., Zombori, G., Rodionov, R., Zuluaga, M.A., Diehl, B., Wehner, T., Miserocchi, A., McEvoy, A.W., Duncan, J.S., Ourselin, S.: Efficient anatomy driven automated multiple trajectory planning for intracranial electrode implantation. In: International Conference on Medical Image Computing and Computer-Assisted Intervention, pp. 542550. Springer (2016)

33. Zelmann, R., Beriault, S., Marinho, M., Mok, K., Hall, J., Guizard, N., Haegelen, C., Olivier, A., Pike, G., Collins, D.: Improving recorded volume in mesial temporal lobe by optimizing stereotactic intracranial electrode implantation planning. International journal of computer assisted radiology and surgery 10(10), 1599-1615 (2015) 